\title{
SKIN COLOUR SEGMENTATION USING FINITE BIVARIATE PEARSONIAN TYPE-IIb MIXTURE MODEL AND K-MEANS
}

\author{
B.N.Jagadesh ${ }^{1}$ K.Srinivasa Rao $^{2}$ Ch.Satyanarayana ${ }^{3}$ G.V.S.RajKumar ${ }^{4}$ \\ ${ }^{1}$ Department of CSE, Srinivasa Institute of Engineering\& Technology, Cheyyeru, \\ Amalapuram, INDIA. \\ nagajagadeshegmail.com \\ ${ }^{2}$ Department of Statistics, Andhra University, Visakhapatnam, INDIA \\ ksraoaudyahoo.co.in \\ ${ }^{3}$ Department of CSE, JNTUK, Kakinada, INDIA \\ chsatyanarayana@yahoo.com \\ ${ }^{4}$ Department of IT, GITAM University, Visakhapatnam, INDIA \\ gvsrajkumar@gmail.com
}

\begin{abstract}
Skin colour segmentation plays an important role in computer vision, face detection and human related systems. Much work has been reported in literature regarding skin colour detection using Gaussian mixture model. The Gaussian mixture model has certain limitations regarding the assumptions like pixels in each component are mesokurtic, having negative range and it doesn't adequately represent the variance of the skin distribution under illumination conditions. In this paper we develop and analyze a new skin colour segmentation based on HSI colour space using bivariate Pearsonian type-IIb mixture model. The model parameters are estimated by deriving the updated equation of EM-Algorithm. The initialization of the model parameters is done through K-means algorithm and method of moments. The segmentation algorithm is obtained using component maximum likelihood under Bayes frame. The experimental results using hue and saturation as feature vector revealed that the developed method perform better with respect to segmentation performance metrics than that of Gaussian mixture model. This method is useful in face detection and medical diagnostics.
\end{abstract}

\section{KEYWORDS:}

Skin segmentation, bivariate Pearsonian type-IIb mixture model, EM-Algorithm, HSI Colour space.

\section{INTRODUCTION}

Recently tremendous amount of effort has been spent on skin colour modeling and detection methods by several authors. Kakumanu et al.[1] have reviewed the articles on skin colour modeling and detection methods and mentioned that the skin colour detection plays an important role in a wide variety of applications ranging from face detection, face tracking, gesture analysis and content based image retrievals to various human computer interaction domains. Skin segmentation deals with detecting human skin areas in an image. It is considered as an important

DOI : 10.5121/sipij.2012.3404 
Signal \& Image Processing : An International Journal (SIPIJ) Vol.3, No.4, August 2012

process for face detection, face tracking and CBIR [2]. Skin colour can also be used as complementary information to other features and can be used to build accurate face detection system $[3,4,5,6]$.

The feature for the detection of skin region is by skin colour so the colour spaces provide the basic framework for feature vector extraction in skin colour detection or segmentation [7, 8, 9]. Different colour spaces like RGB, Normalized RGB, HSV, YCbCr and CIE L*a*b etc., are used for developing the skin colour detections $[10,11,12]$. Choice of colour space is the main aspect of skin colour feature extraction.

Colour space is a multi dimensional space where different components of colours are being represented with different dimensions. Although RGB is the most commonly used colour space unfortunately, it is not perceptually uniform. In RGB space, equal distances in different intensities ranges and along different dimensions of the three dimensional RGB colour space do not correspond to equal perception of colour dissimilarity [13]. Therefore, alternative colour spaces based on RGB colour space transformation are often used. The ideal colour space transformation is to develop a model of a colour space that is perceptually similar with human colour vision.

We can generate colour spaces such as HSI, CIE-Lab and CIE-Luv by nonlinear transformation of the RGB space. Hue, Lightness and Saturation are three characteristics of the CIE colour spaces that best characterize colour space perceptually. The HSI offers the advantages that separate channels outline certain colour properties namely, Intensity (I), Hue (H) and Saturation (S). This specific colour space is often chosen because the visual cognitive system of human beings tends to treat these three components as roughly orthogonal perceptual axis [14]. It decouples the intensity component from the colour carrying information (Hue and Saturation) in the colour visible images. This allows us to treat achromatic and chromatic channels separately since Hue and Saturation are functions of pixel intensity. Rafel C et al. [15] have stated that HSI model is the ideal for digital image processing. Because it is closely related to the way in which people describe the perception of colour. Hence in this paper we develop the skin colour segmentation method by considering that the bivariate feature vector consists of hue and saturation.

In skin colour segmentation it is established that model based methods are more efficient than histogram or edge based methods [16, 17, 18, 19, 20, 21 and 22]. In general the SGM or GMM are used for modeling the skin colour [21]. That is in many of the model based skin colour segmentation methods it is customary to consider that the feature vector of the skin pixels follows a Gaussian distribution. The Gaussian distribution serves well only when the feature vector of the colour region are mesokurtic and symmetric. In many practical situations the feature vector of the colour region may not be mesokurtic and symmetric. As a result of it in some images the results are not accurate.

Hence, in this paper we develop and analyze a skin colour segmentation algorithm with the assumption that the feature vector (consists of hue and saturation of the skin or non-skin regions) follows a bivariate Pearson type-IIb mixture model. The bivariate Pearson type-IIb distribution is a more versatile distribution and includes different shapes of the frequency curves associated with asymmetric/lepti/platykurtic distributions. The bivariate Pearson type-IIb distribution will also 
Signal \& Image Processing : An International Journal (SIPIJ) Vol.3, No.4, August 2012

take care about the correlation between hue and saturation and the non negative nature of these two features.

Here the whole image is characterized by two component mixture of bivariate Pearson type-IIb given by Kotz et al. [23]. The model parameters are estimated by deriving the updated equations of EM-Algorithm. The initialization of the parameters is done through K-means Algorithm and method of moments. A skin colour segmentation algorithm is developed with component likelihood under Bayesian frame. The efficiency of the proposed algorithm is studied by conducting the experiments by taking random sample of five images from JNTUK database and UCD Colour Face Image database and computing the performance measures like confusion matrix, precession, recall and F-ratio. A comparative study is carried by computing the segmentation performance metrics with GMM. The segmentation performance metrics PRI, VOC and GCE for both the algorithms are based on bivariate Pearson type-IIb mixture model and Gaussian mixture model. The developed algorithm is useful for face detection, face tracking and other human interaction domain applications.

\section{BIVARIATE PEARson TYPE - IIb MIXTURE MODEL}

In skin colour analysis the classification of the image is done into two categories namely, skin and non-skin colour regions. The skin colour is different from the colour of most other natural objects in the world. To build the statistical model for the pixels in the image, the feature vector is extracted using colour spaces. In skin colour segmentation one has to use the chrominance component in extracting the features. Accordingly the hue and saturation under HSI colour space are used for skin colour detection. The statistical observations of hue and saturation which form a bivariate feature vector match closely with the bivariate Pearson type-IIb distributions. The bivariate Pearson type-IIb given by Kotz et al. [23] is having non negative and asymmetric nature of the random variable. It also includes a wide variety of bivariate probability distributions. Here it is assumed that the feature vector of the pixel in skin or non-skin regions in the image follows a bivariate Pearson type-IIb distribution. The Joint Probability density function of the feature vector is

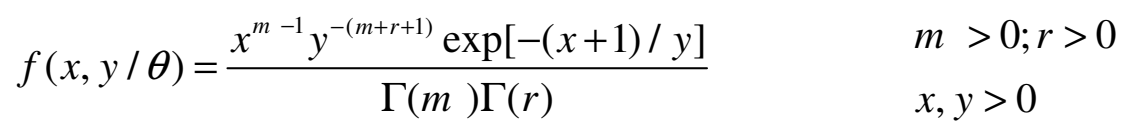

$\theta$ is the parametric set such that $\theta=(m, r)$, x denote the hue value and y denote the saturation value of the pixel in the image.

The marginal probability density function of the hue value is

$$
f(x)=\frac{x^{m-1}}{\beta(m, r)(1+x)^{m+r}} \quad y>0, r, m>0
$$

Its mean is $\frac{m}{(r-1)}$ 
Its variance is $\frac{(r-1) m+m^{2}}{(r-1)^{2}(r-2)}$

The marginal probability density distribution of the saturation value is

$$
f(y)=\frac{1}{\Gamma(r)} y^{-(r+1)} e^{-\left(\frac{1}{y}\right)} \quad y>0, r>0
$$

Its mean is $(r-1)^{-1}$

Its variance is $\frac{r(r-2)}{(r-1)^{2}}$

The Covariance between hue and saturation is $\frac{m}{(r-1)^{2}(r-2)}$

Since the entire image is a collection of skin and non-skin pixel regions which are characterized by a bivariate Pearson type-IIb distribution, the feature vector associated with the whole image is modeled as a two component bivariate Pearson type-IIb mixture model. Its Joint probability density function is

$$
h(x, y)=\sum_{i=1}^{2} \alpha_{i} f_{i}\left(x, y / \theta_{i}\right)
$$

Where, $0<\alpha_{i}<1$ and $\alpha_{1}+\alpha_{2}=1$ and $f_{i}(x, y)$ is as given equation (1).

\section{Estimation of The Model Parameters Using EM- ALGORITHM}

The likelihood function of bivariate observations $\left(x_{1,} y_{1}\right),\left(x_{2}, y_{2}\right),\left(x_{3}, y_{3}\right), \ldots,\left(x_{N}, y_{N}\right)$ drawn from an image with probability density function

$$
\begin{aligned}
& h(x, y ; \theta)=\sum_{i=1}^{K} \alpha_{i} f_{i}\left(x_{s}, y_{s} ; \theta\right) \\
& L(\theta)=\prod_{s=1}^{N}\left(\sum_{i=1}^{K} \alpha_{i} f_{i}\left(x_{s}, y_{s} ; \theta\right)\right) \\
& =\prod_{s=1}^{N}\left(\sum_{i=1}^{K} \alpha_{i} \frac{x^{m-1} y^{-(m+r+1)} \exp [-(x+1) / y]}{\Gamma(m) \Gamma(r)}\right) \quad \begin{array}{l}
r>0 \\
m>0
\end{array}
\end{aligned}
$$

This implies

$$
\begin{aligned}
& \log L(\theta)=\log \prod_{s=1}^{N}\left(\sum_{i=1}^{K} \alpha_{i} f_{i}\left(x_{s}, y_{s} ; \theta\right)\right) \\
& =\sum_{s=1}^{N} \log \left(\sum_{i=1}^{K} \alpha_{i} f_{i}\left(x_{s}, y_{s} ; \theta\right)\right)
\end{aligned}
$$


Signal \& Image Processing : An International Journal (SIPIJ) Vol.3, No.4, August 2012

The model parameters are estimated by using the Expectation Maximization Algorithm (E.M Algorithm).

The updated equation of the parameter $\alpha_{k}$ is

$$
\begin{gathered}
\alpha_{k}^{(l+1)}=\frac{1}{N} \sum_{s=1}^{N}\left[t_{k}\left(x_{s,} y_{s} ; \theta^{(l)}\right)\right] \text { for } \mathrm{K}=1,2 . \\
=\frac{1}{N} \sum_{s=1}^{N}\left[\frac{\alpha_{k}^{l} f_{k}\left(x_{\left.s, y_{s} ; \theta^{(l)}\right)}\right.}{\sum_{i=1}^{2} \alpha_{i}^{l} f_{i}\left(x_{s,} y_{s} ; \theta^{(l)}\right)}\right]
\end{gathered}
$$

where $f_{k}\left(x_{s,} y_{s} ; \theta^{(l)}\right)$ is as given equation (1).

The updated equation of $m_{k}$ at $(l+1)^{\text {th }}$ iteration is

$\sum_{s=1}^{N} t_{k}\left(x_{s,} y_{s} ; \theta^{(D)}\right) \log \left(x_{s}\right)-\sum_{s=1}^{N} t_{k}\left(x_{s,} y_{s} ; \theta^{(D)}\right) \log \left(y_{s}\right)+\sum_{s=1}^{N} t_{k}\left(x_{s,} y_{s} ; \theta^{(D)}\right) \frac{1}{\Gamma m_{k}}\left(\Gamma m_{k} * \log (\log (e))-\Gamma m_{k}^{*} * P_{s i}\left(m_{k}\right) * \log \left(e^{-m_{k}}\right)=0\right.$

where Psi $\left(m_{k}\right)=\operatorname{digamma}\left(m_{k}\right)$

The updated equation of $r_{k}$ at $(l+1)^{\text {th }}$ iteration is

$\sum_{s=1}^{N} t_{k}\left(x_{s,} y_{s} ; \theta^{(l)}\right) \log \left(y_{s}\right)-\sum_{s=1}^{N} t_{k}\left(x_{s} y_{s} ; \theta^{(l)}\right) \frac{1}{\Gamma r_{k}}\left(\Gamma r_{k} * \log (\log (e))-\Gamma r_{k} * P s i\left(r_{k}\right) * \log \left(e^{-r_{k}}\right)=0\right.$

where Psi $\left(r_{k}\right)=\operatorname{digamma}\left(r_{k}\right)$

Solving equations (7), (8) and (9) iteratively using MATLAB code we get the revised estimates of $\alpha_{k}, m_{k}, r_{k}$ for $\mathrm{K}=1,2$.

\section{Initilization OF MOdel Parameters by K-MEANS}

The efficiency of the EM-Algorithm in estimating the parameters is heavily dependent on the initial estimates of the parameters. The number of mixture components taken for K-means algorithm is two (skin and non-skin), i.e., $\mathrm{K}=2$. Usually the mixing parameter and the region parameter $(\mathrm{m}, \mathrm{r})$ are unknown. A commonly used method in initialization is by drawing a random sample in the entire image data $[24,25]$. This method perform well only when the sample size is large, and the computation time is heavily increased. When the sample size is small it is likely that some small regions may not be sampled. To overcome this problem, we use K-means algorithm [26] to divide the whole image into two homogeneous regions representing skin and non-skin regions. We obtain the initial estimates of the parameters $\mathrm{m}$ and $\mathrm{r}$ for each image region using the method of moment estimators for bivariate Pearson type-IIb distribution and for the parameters $\alpha_{i}$ as $\alpha_{i}=\frac{1}{2}$ for $\mathrm{i}=1,2$. 
Therefore the initial estimates of $m_{k}$ and $r_{k}$ can be obtained by equating the theoretical moments with the sample moments. This implies

$$
\begin{aligned}
& \frac{m_{k}}{r_{k}-1}=\overline{x_{k}} \\
& \frac{1}{r_{k}-1}=\overline{y_{k}}
\end{aligned}
$$

where $\overline{x_{k}}$ is the $k^{\text {th }}$ region sample mean of the Hue.

$\overline{y_{k}}$ is the $k^{\text {th }}$ region sample mean of the Saturation.

Solving the equations (10), (11) we get the initial estimates of the parameters $\left(m_{k}\right.$ and $\left.r_{k}\right)$ for $\mathrm{k}=1,2$. Substituting these values as the initial estimates, we obtain the refined estimates of the parameters by using the EM-Algorithm.

\section{SKIN COLOUR SEgMENTATION ALgORITHM}

After refining the parameters the prime step is skin colour segmentation, by allocating the pixels to the skin or non-skin segments. This operation is performed by segmentation algorithm. The algorithm is described as follows

1. Divide the whole image into two regions using K-means algorithm

2. Obtain the initial estimates of the model parameters using the moment estimators as discussed in section 4 for each region

3. Obtain the refined estimates of the model parameters by using the EM-algorithm with the updated equations given in section 3 .

4. Substitute the estimated parameter values in the image joint probability density function

$$
h(x, y)=\sum_{i=1}^{K} \alpha_{i} f_{i}\left(x, y ; \theta_{i}\right) \text { where } f_{i}\left(x, y / \theta_{i}\right) \text { is as given equation (1). }
$$

5. Segment the pixels as skin colour or non-skin colour pixel using a threshold (t) and the likelihood function such that $L(x / \theta) \geq t$ or $L(x / \theta)<t$ respectively for $0<\mathrm{t}<1$.

The optimal threshold value of $t$ is determined computing true positive and false positive over the segmented regions and plotting the ROC Curve.

\section{EXPERIMENTAL RESUlts AND PERFoRMANCE EVAluATION}

In this section, the performance of the developed skin colour segmentation algorithm is evaluated. For this purpose the skin images are collected from JNTUK database and UCD Colour Face Image database. A random sample of 5 images is taken from both the databases and the feature vector consists of hue and saturation for each pixel of the each image is computed utilizing HSI colour space. In HSI colour space the hue and saturation values are computed from the values of RGB for each pixel in the image using the formula 
Signal \& Image Processing : An International Journal (SIPIJ) Vol.3, No.4, August 2012

$$
\begin{aligned}
\text { Hue }=\mathrm{H} & =\cos ^{-1}\left[\frac{(R-G)+(R-B)}{2 \sqrt{(R-G)^{2}+(R-B)(G-B)}}\right], B \leq G \\
& =2 \prod-\cos ^{-1}\left[\frac{(R-G)+(R-B)}{2 \sqrt{(R-G)^{2}+(R-B)(G-B)}}\right], B>G
\end{aligned}
$$

Saturation $=\mathrm{S}=\frac{1-\min (R, G, B)}{I}$

where $\mathrm{I}=\frac{R+G+B}{3}$ is the intensity of pixel.

With the feature vector $(\mathrm{H}, \mathrm{S})$ each image is modeled by using the two component bivariate Pearson type-IIb mixture distribution. The initial values of the model parameters $\alpha_{1}, \alpha_{2}, m_{1}, m_{2}, r_{1}$ and $r_{2}$ are obtained by dividing all the pixels in to two categories namely skin and non-skin region using $\mathrm{K}$-means algorithm with $\mathrm{K}=2$, and taking $\alpha_{1}=\alpha_{2}=\frac{1}{2}$ and moment estimates for $\left(m_{i}, r_{i}\right), \mathrm{i}=1,2$. Using these initial estimates and the updated equations of the EMAlgorithm discussed in section. 3 with MATLAB code the refined estimates of model parameters are obtained. Substituting the refined estimates in the bivariate Pearson type-IIb joint probability distribution function the skin colour and non-skin colour models of each image are estimated. The segmentation algorithm with component maximum likelihood under Bayesian frame and a threshold value $t$ as discussed in section 5 is used to segment the image. Figure. 1 shows the

\begin{tabular}{|c|c|c|c|c|c|}
\hline Images & $\begin{array}{l}\text { Image } 1 \\
\text { (female) }\end{array}$ & $\begin{array}{c}\text { Image } 2 \\
\text { (Baby) }\end{array}$ & $\begin{array}{l}\text { Image } 3 \\
\text { (Group) }\end{array}$ & $\begin{array}{c}\text { Image } 4 \\
\text { (Male) }\end{array}$ & $\begin{array}{l}\text { Image } 5 \\
\text { (Boys) }\end{array}$ \\
\hline Original & & & & & \\
\hline Segmented & & & & & \\
\hline
\end{tabular}
original and segmented images.

Figure.1: Original and segmented sample images 
Signal \& Image Processing : An International Journal (SIPIJ) Vol.3, No.4, August 2012

The developed algorithm performance is evaluated by comparing skin colour segmentation algorithm with the Gaussian mixture model. Table 1. present the miss classification rate of the skin pixels of the sample image using proposed model and Gaussian mixture model.

Table 1. Miss classification rate of the classifier

\begin{tabular}{|c|c|}
\hline Model & Miss Classification Rate \\
\hline BPTIIbMM & $9 \%$ \\
\hline GMM & $12 \%$ \\
\hline
\end{tabular}

From the Table 1. it is observed that the misclassification rate of the classifier with bivariate Pearson type-IIb mixture model( BPTIIbMM ) is less compared to that of GMM.

The accuracy of the classifier is also studied for the sample images by using confusion matrix for skin and non-skin regions. Table 2. shows the values of TPR, FPR, Precision, Recall and Fmeasure for skin and non-skin segments of the sample images.

Table 2. Comparative study of GMM and BPTIIbMM

\begin{tabular}{|c|c|c|c|c|c|c|}
\hline Image & Method & TPR & FPR & Precision & Recall & F-measure \\
\hline \multirow{2}{*}{$\begin{array}{l}\text { Image1 } \\
\text { (Female) }\end{array}$} & ВPTIIbMM & 0.9166 & 0.1250 & 0.9361 & 0.9166 & 0.9262 \\
\hline & GMM & 0.8750 & 0.1830 & 0.9051 & 0.8750 & 0.8898 \\
\hline \multirow{2}{*}{$\begin{array}{l}\text { Image2 } \\
\text { (Baby) }\end{array}$} & BPTIIbMM & 0.9423 & 0.110 & 0.9570 & 0.9423 & 0.9496 \\
\hline & GMM & 0.8846 & 0.300 & 0.8949 & 0.8846 & 0.8947 \\
\hline \multirow{2}{*}{$\begin{array}{l}\text { Image3 } \\
\text { (Group) }\end{array}$} & BPTIIbMM & 0.8928 & 0.1000 & 0.8620 & 0.8928 & 0.8771 \\
\hline & GMM & 0.8214 & 0.1818 & 0.7914 & 0.8214 & 0.8061 \\
\hline \multirow{2}{*}{$\begin{array}{l}\text { Image4 } \\
\text { (Male) }\end{array}$} & ВРТIIbMM & 0.9000 & 0.0576 & 0.8571 & 0.9000 & 0.8780 \\
\hline & GMM & 0.7800 & 0.1615 & 0.6500 & 0.7800 & 0.7090 \\
\hline \multirow{2}{*}{$\begin{array}{l}\text { Image } 5 \\
\text { (Boys) }\end{array}$} & BPTIIbMM & 0.8750 & 0.125 & 0.7777 & 0.8750 & 0.8235 \\
\hline & GMM & 0.7500 & 0.1666 & 0.7010 & 0.7500 & 0.7246 \\
\hline
\end{tabular}

From Table 2. it is obtained that the F-measure value for the proposed classifier is more. This indicates the proposed classifier perform better than that of Gaussian mixture model. Figure. 2 shows the ROC curves associated with the proposed skin colour classifier and the classifier with GMM. 
Signal \& Image Processing : An International Journal (SIPIJ) Vol.3, No.4, August 2012

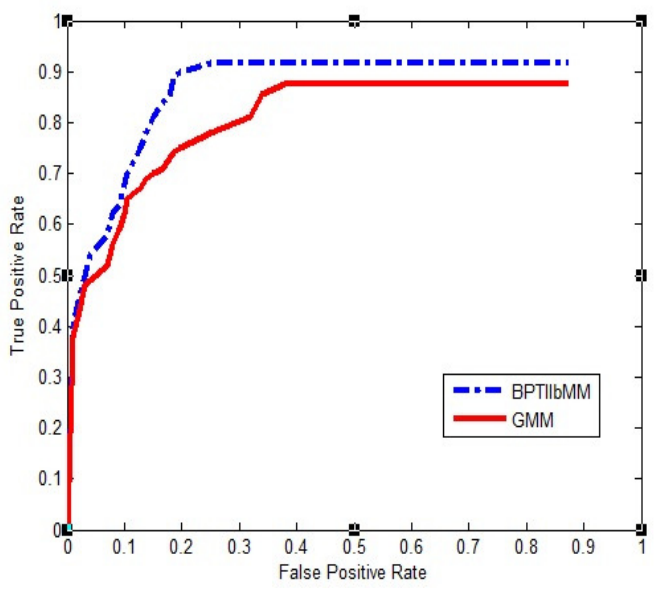

Image 1(Female)

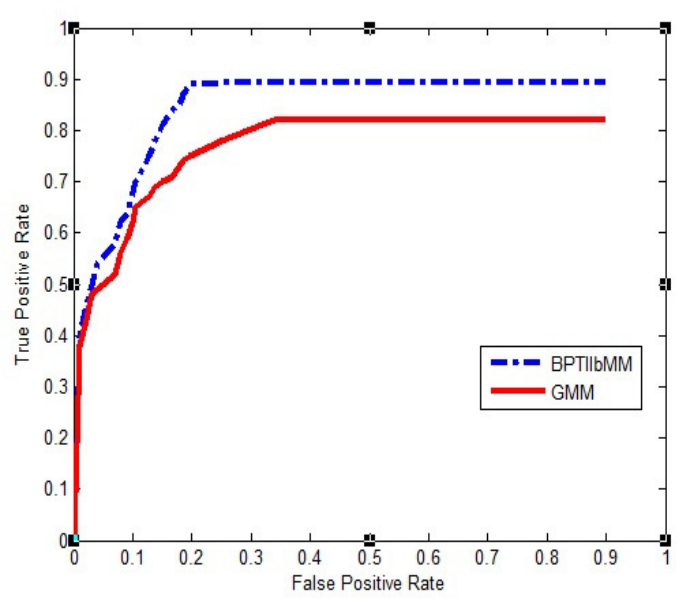

Image 3(Group)

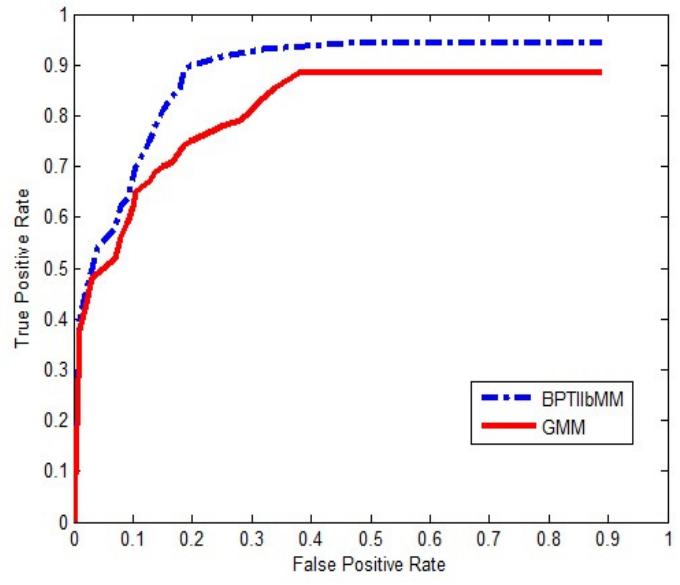

Image 2(Baby)

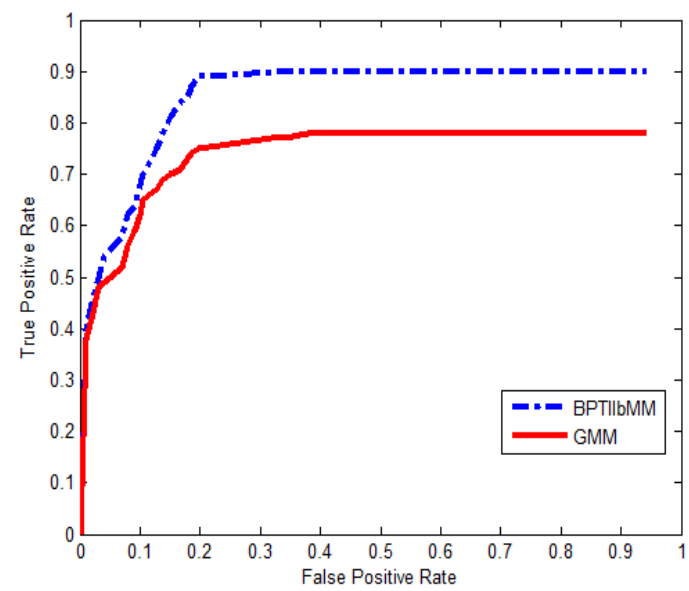

Image 4(Male)

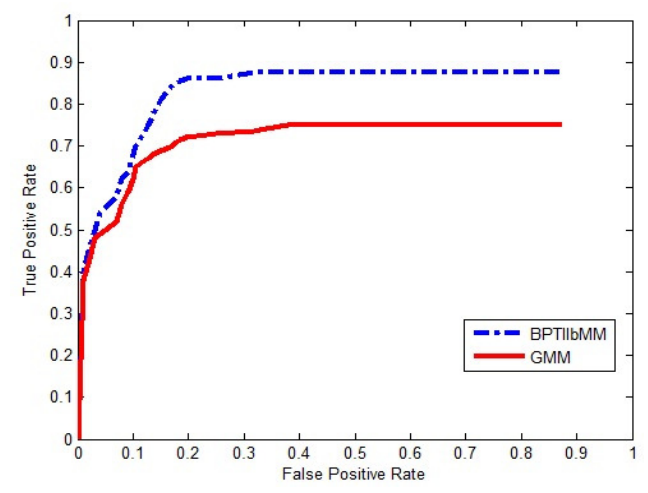

Image 5(Boys)

Figure.2 ROC Curves 
Signal \& Image Processing : An International Journal (SIPIJ) Vol.3, No.4, August 2012

From the Figure. 2 it is observed that the proposed classifier is having less false detection of the skin pixels compared to the classifier with GMM. The figure also shows that can successfully identified the exposed skin region including face, hands and neck.

The performance of the segmentation algorithm is also studied by obtaining three segmentation performance measures namely, Probabilistic Rand Index (PRI) [27], Variation of Information (VOI) [28], Global Consistency Error (GCE) [29] with the sample images. The computed values of the performance measures for the developed algorithm with BPTIIbMM and GMM are presented in Table. 3 .

Table 3.Segmentation Performance Measures

\begin{tabular}{|c|c|l|l|l|}
\hline \multirow{2}{*}{ Image } & \multirow{2}{*}{ Method } & \multicolumn{3}{|c|}{ Performance Measures } \\
\cline { 2 - 5 } & & PRI & GCE & VOI \\
\hline \multirow{2}{*}{$\begin{array}{c}\text { Image 1 } \\
\text { (Female) }\end{array}$} & BPTIIbMM & 0.5313 & 0.3712 & 0.0265 \\
\cline { 2 - 5 } & GMM & 0.4928 & 0.3923 & 0.1286 \\
\hline \multirow{2}{*}{$\begin{array}{c}\text { Image 2 } \\
\text { (Baby) }\end{array}$} & BPTIIbMM & 0.6404 & 0.0124 & 0.0786 \\
\hline \multirow{2}{*}{$\begin{array}{c}\text { Image 3 } \\
\text { (Group) }\end{array}$} & GMM & 0.4627 & 0.1656 & 0.1428 \\
\hline \multirow{2}{*}{$\begin{array}{c}\text { Image 4 } \\
\text { (Male) }\end{array}$} & BPTIIbMM & 0.5125 & 0.0406 & 0.1101 \\
\cline { 2 - 5 } & GPTIIbMM & 0.3984 & 0.1927 & 0.2010 \\
\hline Image 5 & GMM & 0.9854 & 0.0025 & 0.0627 \\
\cline { 2 - 5 }$($ Boys $)$ & BPTIIbMM & 0.6224 & 0.0346 & 0.1402 \\
\cline { 2 - 5 } & GMM & 0.4589 & 0.1724 & 0.2210 \\
\hline
\end{tabular}

From the Table. 3 it is observed the PRI value of the proposed algorithm for sample images considered for experimentation are more than that of the value from the segmented algorithm based on GMM and they are closed to 1. Similarly the GCE and VOI values of the proposed algorithm are less than that of finite Gaussian mixture model and closed to 0 . This reveals that the proposed segmentation algorithm performs better than the algorithm with GMM and the skin colour segmentation is closed to the ground truth. 
Signal \& Image Processing : An International Journal (SIPIJ) Vol.3, No.4, August 2012

\section{CONCLUSION}

A new and novel skin colour segmentation based on bivariate Pearson type-IIb mixture model with K-means is presented. In this approach the pixels of the skin and non-skin regions in the image are characterized by hue and saturation values. The bivariate Pearson type-IIb distribution used for skin region is capable of identifying the skin regions more effectively since it consider the non-negative nature of the feature vector and correlation between hue and saturation values in the colour space. The model parameters are estimated by deriving the updated equation of the EM-Algorithm for the bivariate Pearsonian type-IIb mixture model. Experimentation is carried with five images randomly selected from the database having skin and non-skin regions. A comparative study of the performance measures like the misclassification rate, ROC curves, Precession, Sensitivity and F-ratio for the proposed method and the segmentation with GMM reveals that the proposed method perform better in skin colour identification than that of the Gaussian mixture model. The segmentation performance measures of the image like PRI, VOI and GCE shown that the proposed method segments the image more close to the ground truth. It is also observed that the computational time of this method is less compared to the other model based classifier since it involves only two parameters $(\mathrm{m}, \mathrm{r})$. This method can also work well under different illumination conditions since the hue and saturation values are capable of removing the luminance in the space.

\section{REFERENCES}

[1] P.Kakumanu, S.Makrogiannis, N. Bourbakis (2007) “ A survey of skin-color modeling and detection methods”, Pattern Recognition, vol.40, pp.1106-1122, 2007.

[2] S.El Fkihi, M.Daoudi, D.Aboutajdine (2009) " Skin and Non-Skin probability approximation based on discriminative tree distribution”, ICIP, pp. 2377-2380.

[3] R. Chellappa, C. Wilson, S.Sirohey (1995), “ Human and machine recognition of faces: a survey, Proc. IEEE 83(5), 705-740.

[4] E.Hjelmas, B.K.Low (2001), “Face detection: a survey”, J.Comput.Vision Image Understanding 83 ,236-274.

[5] M.H.Yang, D.J.Kriegman, N.Ahuja (2002), “ Detecting faces in images: a survey", IEEE Trans. Pattern Anal. Mach. Intell. 24 (1), 34-58.

[6] W.Zhao, R.Chellappa, P.J.Philips, A. Rosenfeld (2003), "Face recognition: a literature survey", ACM Comput. Surveys 85(4) 299-458.

[7] Wei Ren Tan, Chee Seng Chan, Pratheepan Yogarajah, and Joan Condell (2012), "A Fusion Approach for Efficient Human Skin Detection", IEEE Transactions on Industrial Informatics, Vol.8, No.1,pp.138-147.

[8] Chang-Yul Kim, Oh-Jin Kwon, and Seokrim Choi (2011), “ A Practical System for Detecting Obscene Videos",IEEE Transactions on Consumer Electronics, Vol.57, No.2, pp. 646-650.

[9] Fumihito Yasuma, Tomoo Mitsunaga, Daisuke Iso, and Shree K. Nayar (2010),“ Generalized Assorted Pixel Camera: Postcapture Control of Resolution, Dynamic Range and Spectrum”, IEEE Transactions on Image Processing, Vol.19, No.9,pp.2241-2253. 
Signal \& Image Processing : An International Journal (SIPIJ) Vol.3, No.4, August 2012

[10] Jalal A.Nasiri, H. Sadoghi Yazdi and Mohmoud Naghibzadeh (2008), "A Mouth Detection Approach Based on PSO Rue Mining on Color Images", 5th Iranian Conference on Machine Vision and Image Processing, November 4-6.

[11] Nils Janssen and Neil Robertson (2008), "On the detection of low-resolution skin regions in surveillance images", The Eighth International Workshop on Visual Surveillance.

[12] Abbas Cheddad, Joan Condell, Kevin Curran and Paul Mc Kevitt (2009), “A New Color space for skin tone detection", ICIP, pp.497-500.

[13] Kato Z, Pang Jing-Chuen (2006), "A Markov random field image segmentation model for color textured images", Image and Computing Vision, Vol.24(10), pp.1103-1114.

[14] G.V.S. Raj Kumar, K.Srinivasa Rao and P.Srinivasa Rao (2011), "Image Segmentation and Retrivals based on finite doubly truncated bivariate Gaussian mixture model and K-means", International Journal of Computer Applications, Volume 25. No.5 pp.5-13.

[15] Rafel C Gonzalez and Richard E Woods (2001), "Digital Image Processing", a text book from Pearson education, India.

[16] J. Yang, W. Lu, and A (1998), Waibel, "Skin-Color Modelling and Adaptation" Proc. ACCV.

[17] B. Menser and F. Muller (1999), "Face Detection in Color Images Using Principal Component Analysis", Proc. Image Processing and its Applications.

[18] L. Fan and K. K. Sung (2000), "Face Detection and Pose Alignment Using Colour, Shape and Texture Information”, Proc. Visual Surveillance.

[19] Y. Raja, S.J. McKenna, and S. Gong (1998) , "Tracking and Segmenting People in Varying Lighting Conditions Using Colour", Proc. Automatic Face and Gesture Recognition.

[20] T.S Jebara and A. Petland (1997), "Parameterized Structure from Motion for 3D Adaptive Feedback Tracking of Faces", Proc. CVPR.

[21] Tiberio S. Caetano, Silvia D. Olabarriaga, Dante A.C.Barone (2003), "Do mixture models in chromaticity space improve skin detection?", Pattern Recognition 36, 3019 - 3021.

[22] Jianyong Sun, Ata Kabán b, Jonathan M. Garibaldi(2010), "Robust mixture clustering using Pearson type VII distribution", Pattern Recognition letters.

[23] Norman L. Johnson, Samuel Kotz and Balakrishnan (2000), "Continuous Multivariate Distributions", John Wiley and Sons Publications, New York.

[24] Mclanchlan G. and Krishnan T. (1997), "The EM Algorithm and Extensions”, John Wiley and Sons, New York -1997.

[25] Mclanchlan G. and Peel D.(2000), “ The EM Algorithm For Parameter Estimations”, John Wileyand Sons, New York - 2000.

[26] Rose H.Turi (2001), "Cluster Based Image Segmentation”, phd Thesis, Monash University, Australia. 
Signal \& Image Processing : An International Journal (SIPIJ) Vol.3, No.4, August 2012

[27] Unnikrishnan R., Pantofaru C., and Hernbert M. (2007), "Toward objective evaluation of image segmentation algorithms,” IEEE Trans.Pattern Annl.Mach.Intell, Vol.29 ,No.6, pp. 929944.

[28] Meila M. (2005), "Comparing Clustering - An axiomatic view," in proc.22nd Int. Conf. Machine Learning, pp. 577-584.

[29] Martin D. Fowlkes C., Tal D. and Malik J. (2001), “ A database of human segmented natural images and its application to evaluating segmentation algorithms and measuring ecological statistics," in proc. 8th Int. Conference Computer vision, vol.2, pp.416- 423.

\section{Authors}

Mr.B.N.Jagadesh is presently working as Assistant Professor in Computer science and Engineering Department at Srinivasa Institute of Engineering \& Technology, Cheyyeru. He presented several research papers in national and International conferences and seminars. He published a good number of papers in national and International journals. He guided several students for getting their M.Tech degrees in Computer Science \& Engineering. His current research interests are Image Processing, Speech Processing and Data Mining.

Prof. K.Srinivasa Rao is presently working as Professor and Head, Department of Statistics, Andhra University, Visakhapatnam. He is elected chief editor of Journal of ISPS and elected VicePresident of Operation Research of India. He guided 28 students for Ph.D in Statistics, Mathematics, Computer Science, Electronics and Communications Engineering, Industrial Engineering and Operations Research. He published 90 research papers in national and International journals with high reputation. His research interests are Image Processing, Communication Systems, Data Mining and stochastic models.

Dr. Ch. Satyanarayana is Associate Professor in Computer science and Engineering Department at Jawaharlal Nehru Technological University Kakinada. He has 13 years of experience. His area of interest is on Image processing, Database Management Systems, Speech Recognition, Pattern recognition and network security. He published more than 20 research papers in international journals. He published more than 30 research papers in international conferences.

Mr. GVS RAJKUMAR is presently working as Associate professor in the Department of Information Technology, GITAM University, Visakhapatnam. He presented several research papers in national and International conferences and seminars. He published a good number of papers in national and International journals. He guided several students for getting their M.Tech degrees in Information Technology. His current research interests are Image Processing, Internet Technologies and Network security. 